\title{
Schedule of Factors Influencing Adherence (SOFIA) to Psychiatric Treatment in Persons with Schizophrenia: Validity and Pilot Testing
}

Shanivaram Reddy $\mathrm{K}^{*}, \quad \mathrm{~N}$ Krishna Reddy', Jagadisha Thirthalli ${ }^{\ddagger}$, C Naveen Kumar§, N R Renukadevi**, Vikram

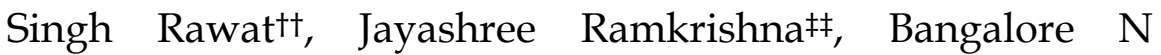
Gangadhar§§

\section{Abstract}

Qualitative research has highlighted the complex interplay of multiple factors that preclude persons with schizophrenia in rural Indian settings for discontinued psychiatric treatment. In this context, this paper aims to establish the face and content validities of an interview schedule titled 'Schedule of Factors Influencing Adherence (SOFIA) to Psychiatric Treatment in Persons with Schizophrenia' which comprehensively assesses factors for discontinued psychiatric treatment and the feasibility of its administration of the schedule. SOFIA contains 16 factors. This schedule involves three phases of

\footnotetext{
Department of Social Work, Jnanabharathi Campus, Bangalore University, Bangalore-560049; shanivaramreddyk@gmail.com (Corresponding author)

† Additional Professor of Psychiatric Social Work, National Institute of Mental Health and Neurosciences (NIMHANS), Hosur Road, Bangalore560029

‡ Additional Professor of Psychiatry, (NIMHANS)

$\S$ Assistant Professor of Psychiatry, NIMHANS)

** Medical officer in the Department of Psychiatry, NIMHANS)

t† Senior Resident Department of Psychiatry, NIMHANS)

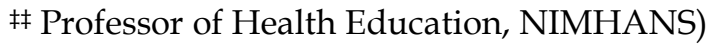

$\S \S$ Professor of Psychiatry, (NIMHANS)
} 
interviewing patients and family members. This was given to twelve experts who used likert scales to rate each items wells as the dimensions of the schedule. Later on, fifteen persons with schizophrenia were interviewed with SOFIA to test the feasibility of administration. The results showed that Fourteen items were rated as either satisfactory $($ score $=4)$ or very much satisfactory $($ score $=5$ ) by all twelve experts; remaining two were rated as 4 or 5 by 11 experts. Regarding comprehensiveness of the factors, scoring methods and general instructions given to the interviewers, all provided scores $>4$; regarding method of interviewing, 11 provided score of $>4$; with regard to overall interview schedule, all experts provided scores $>4$. Pilot testing revealed that it took 60 minutes to administer SOFIA. SOFIA has satisfactory face and

Keywords: Factors of adherence, Psychiatric care, Face validity, Content validity

\section{Introduction}

Schizophrenia is a chronic mental illness that impairs a person's ability to think, feel, and express self. It generally results in reduced functional capacity, poor quality of life and low chances of remission (Thara et al,. 2008) but substantial proportions of schizophrenia patients discontinued psychiatric treatment in developing countries (WHO, 2001). There are several factors such as cost, scarcity of community and hospital based services, stigma, and shortages in the workforce etc associated with poor compliance to psychiatric treatment. However, the reason for this phenomenon is not well understood even in developed countries (Ascher et al, .2010, Thara et al . 2008).

In India, research in the field of continued psychiatric treatment among mentally ill persons in a community setting is still in its infancy. Murthy et al. (1974) pointed out that there are no reports studying this problem in depth in Indian setting to provide guidelines to modify patient behaviour. On the whole, the research on the social aspects of continued psychiatric treatment is yet to take birth that too in the community setting. Especially needed is 
the in-depth study of treatment adherence with big samples (Parthasarathy et al; 1981).

Most Indian studies have used checklists (Barrowclough et al,.1987, Naik et al ,.1996, Boral et al,. 1980) to assess the reasons for discontinued psychiatric treatment. Since the problem of discontinued psychiatric treatment is a complex one, use of prepared checklists and interviewer-directed questionnaires are poorly suited to understand the issue comprehensively. They often fail to capture many patient / family related factors. Further, these checklists/interviews are not standardized. Comprehensive, qualitative studies are ideally suited to understand these issues. We have developed a comprehensive tool to assess causes for discontinued psychiatric treatment in patients with schizophrenia [Schedule of Factors Influencing Adherence (SOFIA) to Psychiatric Treatment in Patients with Schizophrenia]. This paper establishes the face and content validities of SOFIA.

\section{Materials and methods}

The participants came from a community intervention program titled 'Treating Untreated Psychosis in Rural Community: Variation in the Experience of Care (TURUVECARE)' entailing identification treatment and follow-up of all schizophrenia patients in Turuvekere, a south Indian Taluk (an administrative block). The taluk has a population of 174,297 according to census 2001.[11]

\section{Identification of patients}

The project team trained 12 primary care doctors and 361 health workers (from all cadres) in applying a simple tool titled 'Symptoms in others'.[12] This is a validated instrument for identifying psychiatric disorders in the community, which coulb be used by grass-root level health staff. They were asked to refer all such patients to the study team. In addition, the research social workers visited each village with the purpose of case finding. They interviewed key informants in the community. After identifying patients, enquiries were made about similar persons that they may have come across. After complete description of the study to the participants/family members, written informed consent was 
obtained. The data was collected between December 2009 and April 2010.

\section{Assessments}

\section{Recruitment and diagnosis}

Diagnosis was made by psychiatrists using the Mini- International Neuropsychiatric Interview (MINI).[13] Their psychopathology and disability were assessed using the positive and negative syndrome scale ${ }^{[14]}$ and the Indian Disability Evaluation and Assessment Scale (IDEAS), [15] respectively. Consecutive patients with history of having never received psychiatric treatment were recruited till we achieved 'saturation' of factors (see below; $n=16$ ). One patient on any given day was recruited if two patients were identified on the same day. However, only the first patient was assessed, as interviewing two patients and their families posed practical challenges in terms of time. 'Psychiatric treatment' was defined as evaluation and treatment by doctors trained in modern allopathic treatment. A total of 716 individuals were referred to or identified by the study team. Among these, 137 had a diagnosis of schizophrenia; 27 suspected patients, were not assessed because they were too symptomatic to provide valid consent and the research team could not trace their family members. Among the 137, $40(29.2 \%)$ were never treated, $63(46 \%)$ were not on treatment at the time of recruitment but had received psychiatric treatment at least once in the past and 34 (24.8\%) were on continuous treatment.

Patients and their family members were interviewed. Interviewing severely symptomatic patients was difficult. "Family members" were defined as caregivers above 18 years, living under the same roof as that of the patient at least during the past year and being responsible for the patient's overall care. Interviews were conducted in the following four phases.

\section{Phase I (10-15 minutes)}

In this phase, they were asked about the following: Possible reasons for not providing psychiatric treatment for their ill relative; how they were managing patient's altered behaviour. This phase was conducted using open-ended questions and family members were 
encouraged to provide as much details as possible using facilitating questions like, "what else?", "then what?", "is there anything else?" etc.

\section{Phase II}

In this phase, semi-directive questions were used to obtain further elaboration on each of the reasons listed above. Examples and illustrations were provided. Discrepancies and contradictions were reflected back.

\section{Phase III}

In this phase, a more directive questioning was conducted using a list of reasons obtained from the existing literature [Table 1] focusing on such reasons not covered so far. Phase-II techniques were used to get elaboration and clarification.

\section{Phase IV}

In this phase, the family members were asked to cite the most and the least important reason (from among the reasons they had already listed), which prevented them from seeing psychiatric treatment.

\section{Data management and analysis}

All interviews were transcribed. Color coding was done to synthesize factors. Representative quotes were selected for each of these factors. The quotes and the colour codes were reviewed by authors independently to reduce biases.

\section{Saturation of factors}

Data collection and interpretation took place simultaneously. Whenever a new reason emerged, it was added to the existing list (that was used in Phase-III). From the $16^{\text {th }}$ patient onwards, no new reasons emerged. Three more families were interviewed to ensure that there were no additional reasons. Thus, families of 19 patients were interviewed.

The study was approved by the ethics committee of National Institute of Mental Health and Neurosciences (NIMHANS). 
Participants were included only after the provision of the written informed consent.

\section{Contents and Administration of SOFIA}

SOFIA covers the following factors: Caregivers' knowledge about and attitude towards mental illness and its treatment, Insight and cooperation from the patient towards treatment, Knowledge about treatment, Perceived beneficial effects of treatment, Adverse effects of medications, Frustration regarding long-term treatment, Gender of the patient, Age of the patient, Financial Factors, Support / supervision from family / well-wishers, Therapeutic relationship with mental health professionals, Treatment-centre related issues, Distance / transport, Logistic Factors, Major life events and Other medical conditions. In addition to the above mentioned factors, the schedule covers all other factors that the patient/family member may bring in. SOFIA is a semi-structured instrument.

Administration involves the following three phases of interviewing.

Phase-1: The interviewer introduced the purpose of the interview and invited responses about factors responsible for discontinued psychiatric treatment. Examples of this open-ended approach would be: 'Could you tell me about the reasons for discontinued psychiatric treatment'; Please tell me about those reasons and / or the difficulties that you faced'. The interviewer used to note down the factors for further inquiry without interrupting the flow of conversation. If the respondent stoped after listing a few, the interviewer encouraged him / her to think about more factors by saying, "anything else?", "go on", etc.

Phase-2: The interviewer sought details regarding each of the factors raised by the patient / caregivers during phase-1. 'You told me that ... is a reason / difficulty. Could you please elaborate on this issue? How did it prevent you from continuation of treatment?

Phase-3: Here the interviewer questioned about the factors that are present in SOFIA, that are not covered during phase- 1 and 2 . The authors have provided few sample questions for each SOFIA factor. To the extent possible, these questions had been framed in such a 
way so as to reflect natural conversation (without using any technical words). The interviewer was encouraged to use these questions. However, he/she was free to use his / her own style to ensure good quality information. If patients / family members started narrating their experience regarding a different unrelated factor, it was suggested not to interrupt them but to score that factor first and then proceed with the previous factor.

\section{General scoring instructions}

Following are the guidelines:

0 . No influence: This factor did not have any influence.

1. Some influence: This factor had some influence. There is reason to believe that though this factor has played some role, other factors have been more influential relative to this.

2. Significant influence: This factor has played a significant role. This may be a key factor that has caused the patient / family to discontinue psychiatric treatment. However, there is reason to believe that if all other factors were conducive, then despite this factor, patient/family would have continued treatment.

3. Profound influence: This factor is the single most important cause for the patient remaining untreated. There is reason to believe that the patient / family would have discontinued treatment even when all other factors were conducive. The influence of this factor may make the assessment of other factors difficult, as it has an overarching effect on all other causes. Thus this scoring has to be used after careful consideration of all other factors. It is expected that the use of this score would be very uncommon.

In general, scoring was done after interviewing the primary caregiver/s. Patients were interviewed based on the level of cooperation from them for a meaningful interview. Interviewing took about 60 minutes. In phases 2 and 3, the interviewer kept anchor points in mind to determine the exact scoring of factors.

\section{Face and Content Validity}

This schedule was given to twelve experts to establish its face and content validities. They used likert scales 1 (Not at all satisfactory), 
2 (somewhat unsatisfactory), 3 (neutral), 4 (satisfactory), 5 (very satisfactory) to rate each item of the schedule. In addition, the experts rated (same way as above) the following five dimensions of the schedule (as a whole) separately: A (comprehensiveness of the factors), B (scoring system), C (interviewing method), D (general instructions given to the raters), $\mathrm{E}$ (overall schedule). The experts were requested to provide specific comments including suggestions to improvise if they rated 1 (Not at all satisfactory), 2 (somewhat unsatisfactory) or 3 (neutral)

\section{Results}

Face and Content Validity: Opinions were sought from 12 experts: 6 faculties from the department of Psychiatry, 3 faculties from department of psychiatric social work, 2 faculties from department of psychology and 1 faculty from department of statistics at the National Institute of Mental Health and Neuro Sciences (NIMHANS), Bangalore. The experts had mean of 15 years and 8.9 years (SD) of post qualification experience of working with patients with severe mental illnesses and their families.

With regard to individual items of SOFIA, 14 were rated as either satisfactory $($ score $=4)$ or very much satisfactory $($ score $=5$ ) by all twelve experts; remaining two factors got a score of $<4$. Regarding comprehensiveness of the factors, scoring methods, general instructions given to the interviewers, all experts provided scores $>$ 4; regarding method of interviewing, 11 experts provided score of $>$ 4; with regard to overall interview schedule, all experts provided scores $>4$.

Feasibility of administering SOFIA: For this purpose, a pilot study was done by administering the instrument on 15 persons with schizophrenia (and their family members) who had discontinued psychiatric treatment. Mean (SD) age of patients was $36.67(12.15)$ years; out of fifteen patients 8(53.3) were females, among them $10(66.7 \%)$ were married, 4 (26.7) unmarried, mean (SD) family income was Rs 2686(3374.24); mean (SD) patient education was $2.76(1.39)$ years and mean (SD) duration of illness was 106.40 (71.26) months. 
Average time taken to administer the entire schedule was 60 minutes. Factors such as Caregivers' knowledge and attitude towards mental illness and its treatment, Insight and cooperation from the patient towards treatment, Knowledge about treatment, perceived beneficial effects of treatment, adverse effects of medications, Frustration regarding long-term treatment, financial factors, support / supervision from family / well-wishers and distance / transport were moderate or significant influence to continued treatment. Remaining factors such as gender of the patient, age of the patient, support / supervision from family / well-wishers, therapeutic relationship with mental health professionals, logistic factors, major life events and other medical conditions did not seem to influence continuation of the treatment. No one particular factor had a profound influence on continued treatment. For each patient/family, there were more than one factor which had either marginal or significant influence on continued treatment. Details regarding this are provided in table-1

\section{Discussion}

A comprehensive tool that assesses barriers to continued psychiatric treatment is an important need. This study establishes the face and content validity and feasibility of administering one such tool that may fulfil this void. SOFIA has been developed using well established qualitative methodology. Feedback from the experts regarding various dimensions of this tool further adds to its validity. Pilot testing revealed not only its feasibility but also its comprehensiveness; in the sense that no new factor emerged even after interviewing fifteen patients and families.

Scoring guidelines are as follows: it should be scored after interviewing the primary caregiver/s. It is advisable to interview the patients too wherever they are able to cooperate for meaningful interview. There are 3 phases of interview, which would need about 60 minutes. In this schedule, the term 'psychiatric treatment' is used to mean allopathic care provided by a qualified professional, including psychiatrist. Other forms of treatment are not considered as psychiatric treatment insofar as this schedule is concerned. Patients / families may refer to many problems 
unrelated to discontinue psychiatric treatment. The interviewer should listen to these and cross-check whether these influenced their decision to discontinue psychiatric treatment. Each patient / family may have a unique set of reasons for discontinued treatment. In this background, the "total" score will not have any meaning. It is expected that the interviewer has information regarding the patient's / family's basic socio-demographic details, including age, sex, marital status, occupation, other members in his / her household, symptoms and duration of the psychiatric illness etc., before using this schedule. These should be kept in mind while interviewing. Additionally, pilot testing has established the feasibility of administering the schedule. Though one item of the schedule has been detailed in the appendix, full copy of the schedule may be obtained after writing to the corresponding author.

\section{Conclusion}

SOFIA fulfils an important need in schizophrenia research in our country. The tool comprehensively assesses barriers to continue the psychiatric treatment for patients with schizophrenia (and their families) in rural communities. The schedule taps many more factors than a checklist does and shows which factor is more important and which is less in each individual. Doing this may help in prioritizing the public health interventions.

\section{Acknowledgments}

The researchers would like to thank ASHA workers, health workers, and Primary health doctors in Turuvekere community, which helped in the identification of patients in the community. This work was conducted with the help of research grants to Dr Jagadisha Thirthalli from the Government of Karnataka and to Dr C Naveen Kumar from the American Psychiatric Association as part of 'Young Minds in Psychiatry' award (year 2009). 


\section{References}

Ascher, S. H., Nyhuis, A. W., Stauffer, V., Kinon, B. J., Faries, D. E., Phillips, G. A., Schuh, K., Awad, A. G., Keefe, R. \& Naber, D. (2010). Reasons for discontinuation and continuation of antipsychotics in the treatment of schizophrenia from patient and clinician perspectives. Journal of Medical Research Opinion, 10(26), 2403-2410. DOI:10.1185/03007995.2010.515900

Barrowclough, C., Tarrier, N., Watts, S., Vaughan, C., Bamrah, J. S. \& Freeman, H. L. (1987). Knowledge about Schizophrenia Interview. British Journal of Psychiatry, 151-158. DOI:10.1192/bjp.bp

Boral, G. C., Bagchi, R. \& Nandi, D. N. (1980). An opinion survey about the causes and treatment of mental- illness and the social acceptance of the mentally ill patients. Indian Journal Psychiatry, 22, 235-238. DOI: 10.4103/0019-5545.105541

Murthy, R. S., Ghosh, A. \& Wig, N. N. (1974). Treatment Acceptance Patterns in a Psychiatric Out-Patient Clinic: Study of Demographic and Clinical Variables. Indian Journal Psychiatry, 16, 323-329. DOI: 10.1016

Naik, A. N., Parthasarathy, R., \& Isaac, M. K. (1996). Families of rural mentally ill and treatment adherence in district mental health programme. International Journal of Social Psychiatry, 42, 68-72. DOI:10.1001/archpsyc.1974.01760160005001.

Parthasarathy, R., Chandrashekar, C. R., Isaac, M. K. \& Prema, T. P. (1981). A profile of the follow up of the rural mentally ill. Indian Journal Psychiatry, 2; 23,139-141. DOI:10.1186/1750-1172-2

Thara, R., Padmavati, R., Jothy, R., Aynkran, J. R., \& Sujit, J. (2008). Community mental health in India: A rethink: International Journal of Mental Health Systems, 232-236. DOI:10.1186/1471-2393-12-99.

World Health Organization Atlas. (2001). Country Profiles on Mental Health Resources. Geneva, World Health Organization. 
Table 1. Results of the pilot testing

\begin{tabular}{|l|l|l|l|l|}
\hline Factors & $\begin{array}{l}\text { No } \\
\text { influence }\end{array}$ & $\begin{array}{l}\text { Some } \\
\text { influence }\end{array}$ & $\begin{array}{l}\text { Moderate } \\
\text { influence }\end{array}$ & $\begin{array}{l}\text { Significan } \\
\text { t influence }\end{array}$ \\
\hline $\begin{array}{l}\text { Caregivers knowledge about and } \\
\text { attitude towards mental illness and } \\
\text { its treatment }\end{array}$ & $0(0)$ & $3(20)$ & $10(66.7)$ & $2(13.3)$ \\
\hline $\begin{array}{l}\text { Insight and cooperation from the } \\
\text { patient towards treatment }\end{array}$ & $0(0)$ & $0(0)$ & $10(66.7)$ & $5(33.3)$ \\
\hline Knowledge about treatment & $0(0)$ & $0(0)$ & $13(86.7)$ & $2(13.3)$ \\
\hline $\begin{array}{l}\text { Perceived beneficial effects of } \\
\text { treatment }\end{array}$ & $0(0)$ & $1(6.7)$ & $13(86.7)$ & $1(6.7)$ \\
\hline Adverse effects of medications & $0(0)$ & $4(26.7)$ & $8(53.3)$ & $3(20.0)$ \\
\hline $\begin{array}{l}\text { Frustration regarding long-term } \\
\text { treatment }\end{array}$ & $0(0)$ & $1(6.7)$ & $9(60.0)$ & $5(33.3)$ \\
\hline Gender of the patient & $10(66.7)$ & $1(6.7)$ & $4(26.7)$ & $0(0)$ \\
\hline Age of the patient & $11(73.3)$ & $1(6.7)$ & $3(20.0)$ & $0(0)$ \\
\hline Financial Factors & $4(26.7)$ & $2(13.3)$ & $8(53.3)$ & $1(6.7)$ \\
\hline $\begin{array}{l}\text { Support / supervision from family / } \\
\text { well-wishers }\end{array}$ & $2(13.3)$ & $5(33.3)$ & $5(33.3)$ & $3(20.0)$ \\
\hline $\begin{array}{l}\text { Therapeutic relationship } \\
\text { mental health professionals }\end{array}$ & $8(53.3)$ & $3(20.0)$ & $4(26.7)$ & $0(0)$ \\
\hline Treatment-centre related issues & $3(20.0)$ & $6(40.0)$ & $6(40.0)$ & $0(0)$ \\
\hline Distance / transport & $0(0)$ & $3(20)$ & $11(73.3)$ & $1(6.7)$ \\
\hline Logistic Factors & $4(26.7)$ & $6(40.0)$ & $5(33.3)$ & $0(0)$ \\
\hline Major life events & $9(60.0)$ & $5(33.3)$ & $1(6.7)$ & $0(0)$ \\
\hline Other medical conditions & $14(93.3)$ & $1(6.7)$ & $0(0)$ & $0(0)$ \\
\hline
\end{tabular}

\section{Appendix: 1}

Frustration regarding long-term treatment: Patients and family members may have discontinued psychiatric treatment due to frustration of having to take medications continuously for days on end as the reason some of them report that they are reminded to be "Patients". On the other this may happen even in the context of patients experiencing substantial benefit out of treatment, but this is more likely in those perceiving partial benefit.

0. No adverse influence or positive influence: The patient and family do not cite this as a factor influencing on ever treatment. This may be particularly so because the treatment has begun only recently. 
1. Some adverse influence: The patient and/or family members express burden because of this factor, but there is no actual instance of being non-adherent because of this factor.

2. Moderate adverse influence: There have been actual instances of non-adherence because of this factor, but these have happened in the context of other equally important factors. For example, the patient/family has perceived some benefit of treatment, but is not entirely satisfied with the improvement. These factors could have together caused instances of non-adherence.

3. Significant adverse influence: This factor has been cited as the key factor causing ever treatment. However, there are other factors too and the patient would have been adherent factors were conducive for continued.

4. Profound adverse influence: This factor surpasses other influencing factors playing a significant role in causing for ever treatment. There is reason to believe that even if all other factors were conducive for continued the treatment, the patient / family could have discontinued because of the frustration associated with having to take treatment for a long time. 